\title{
Correction to: Mapping and characterization of wheat stem rust resistance genes SrTm5 and Sr60 from Triticum monococcum
}

\author{
Shisheng Chen ${ }^{1} \cdot$ Yan Guo $^{1,2} \cdot$ Jordan Briggs $^{3} \cdot$ Felix Dubach $^{1} \cdot$ Shiaoman Chao ${ }^{4} \cdot$ Wenjun Zhang ${ }^{1}$. \\ Matthew N. Rouse ${ }^{3,5}$. Jorge Dubcovsky ${ }^{1,6}$ (i)
}

Published online: 11 October 2018

๑) Springer-Verlag GmbH Germany, part of Springer Nature 2018

\section{Correction to: \\ Theoretical and Applied Genetics (2018) 131:625-635 \\ https://doi.org/10.1007/s00122-017-3024-z}

In the original publication, the IWGSC assembly is incorrectly referenced.

The paragraph "Coordinates in the Chinese Spring wheat genome were based on the IWGSC CS WGA v1.0 assembly (Zimin et al. 2017) and those in tetraploid wheat on the NRGene assembly of the T. turgidum subsp. dicoccoides accession Zavitan (Avni et al. 2017)" should read:

"Coordinates in the Chinese Spring wheat genome were based on the IWGSC RefSeq v1.0 (International Wheat Genome Sequencing Consortium 2018) and those in tetraploid wheat on the NRGene assembly of the T. turgidum subsp. dicoccoides accession Zavitan (Avni et al. 2017)."

The correct reference for this reference genome is: International Wheat Genome Sequencing Consortium (2018), Science 361: eaar7191. https://doi.org/10.1126/scien ce.aar7191.

The original article can be found online at https://doi.org/10.1007/ s00122-017-3024-z.

Jorge Dubcovsky

jdubcovsky@ucdavis.edu

1 Department of Plant Sciences, University of California, Davis, CA 95616, USA

2 Department of Plant Pathology, China Agricultural University, Beijing 100193, China

3 Department of Plant Pathology, University of Minnesota, St. Paul, MN 55108, USA

4 USDA-ARS Biosciences Research Laboratory, 1605 Albrecht Blvd. N., Fargo, ND 58102, USA

5 USDA-ARS Cereal Disease Laboratory, St. Paul, MN 55108, USA

6 Howard Hughes Medical Institute, Chevy Chase, MD 20815, USA 